\title{
The effect of carbohydrate ingestion on the experience of fatigue during prolonged exercise
}

\author{
Lonneke Albert \\ Maastricht University \\ lonnekealbert@hotmail.com
}

\section{Abstract}

Background: Muscle fatigue occurs during prolonged exercise as the body's energy stocks in the form of muscle glycogen and blood glucose levels within the body become depleted. The sensation of fatigue can become so intense that the person must reduce his or her efforts, or even stop the exercise altogether. Studies have shown that the ingestion of carbohydrate solutions can delay this onset of fatigue. The ingestion of multiple transportable carbohydrates can improve performance and increases exercise capacity more than single transportable carbohydrates. Therefore, the ingestion of multiple transportable carbohydrates may decrease the experience of fatigue during a fixed workload. The purpose of the randomized, double-blind, placebo-controlled study presented in this paper is to explore the effects of the ingestion of multiple transportable carbohydrates such as glucose with fructose, and glucose with sucrose, in decreasing the experience of fatigue as compared to an isoenergetic amount of glucose. Methods: Twelve healthy, trained male athletes cycled 180 minutes at $50 \%$ maximum power output while receiving a solution providing either $1.8 \mathrm{~g} / \mathrm{min}$ of glucose (GLU), $1.2 \mathrm{~g} / \mathrm{min}$ of glucose + $0.6 \mathrm{~g} / \mathrm{min}$ of fructose (GLU+FRUC), $0.6 \mathrm{~g} / \mathrm{min}$ glucose $+1.2 \mathrm{~g} / \mathrm{min}$ sucrose (GLU+SUC) or water (WAT). The experience of fatigue was assessed with the Borg scale where subjects were asked to rate their perceived exertion every 30 minutes during exercise. Results: The results of this study cannot be published. Conclusion: Although the results cannot be published, some speculations can be made. RPE (ratings of perceived exertion) are expected to differ significantly between the four ingested solutions during the later stages of exercise. The expectation is that the RPE for those subjects ingesting multiple transportable carbohydrates will be lower than those ingesting glucose and water. The same should hold true for the single transportable carbohydrate versus water trials. In summary, when glucose and fructose or glucose and sucrose are ingested simultaneously at high rates during cycling exercise, the experience of fatigue is likely to be reduced in comparison to the ingestion of an isoenergetic amount of glucose. 
Keywords

Ratings of perceived exertion, fatigue, carbohydrate ingestion, carbohydrate oxidation, blood glucose, exercise, cycling.

\section{Introduction}

Muscle fatigue can be defined as the failure to maintain the required force during exercise (1). During prolonged exercise, fatigue occurs as heat is generated and lactate, hydrogen ions, $\mathrm{CO}_{2}$ and ammonia accumulate in the muscles, thereby affecting the equilibrium of the internal environment (2). Furthermore, muscle glycogen and blood glucose levels become depleted (2). The depletion of these energy stocks are important determinants of fatigue during prolonged exercise $(3,4)$. The sensation of fatigue can become so intense that the workload can no longer be maintained.

The onset of fatigue is delayed when carbohydrates are ingested before and during prolonged exercise $(3,5,6)$. Burgess and colleagues (1991) have shown that fatigue is delayed when an $8 \%$ glucose solution ingested during 180 minutes of exercise (3). Fatigue assessed as ratings of perceived exertion (RPE) was attenuated during exercise, likely due to the maintenance of blood glucose, increased carbohydrate oxidation and by sparing muscle glycogen levels following carbohydrate ingestion $(3,7)$. Glycogen levels are low in the latter stages of exercise, but blood glucose can still supply energy to working muscles. Therefore, carbohydrate feeding and thereby the maintenance of blood glucose may delay the onset of fatigue during prolonged exercise. As a result, there appears to be a relationship between high blood glucose levels, high carbohydrate oxidation rates and attenuated RPE during prolonged exercise (3).

Total carbohydrate oxidation and exogenous carbohydrate oxidation can be increased and maintained when carbohydrates are ingested. It is likely that this increase in oxidation rates improves performance and increases exercise capacity (for review see (8)). Especially high exogenous carbohydrate oxidation rates have the potential to improve prolonged exercise performance, as endogenous carbohydrate stores become depleted during exercise (9). There exists a dose-response relationship between carbohydrate ingestion rates, exogenous carbohydrate oxidation rates and performance (10). Ingestion of glucose up to 1.0 to $1.5 \mathrm{~g} / \mathrm{min}$ leads to a peak exogenous carbohydrate oxidation rate of $\sim 1 \mathrm{~g} / \mathrm{min}$ (11). However, there is no further increase in exogenous carbohydrate oxidation rate when glucose is ingested at higher rates $(12,13)$. 
A possible rate limiting factor for exogenous carbohydrate oxidation is intestinal absorption (12). Glucose is transported for absorption by sodium-dependent glucose transporters (SGLT1). When glucose is ingested at a rate higher than $1.2 \mathrm{~g} / \mathrm{min}$, this may not have an additional effect on exogenous carbohydrate oxidation rate because the SGLT1 are likely to become saturated (12). Interestingly, fructose is a carbohydrate that uses a different transporter than glucose. Fructose is transported for absorption via GLUT5 transporters. Furthermore, sucrose is a disaccharide that is hydrolyzed at the brush-border membrane to glucose and fructose in a ratio of 1:1. It is likely that the absorption continues via the same intestine carbohydrate transporters as free glucose and free fructose (14).

The maximum exogenous carbohydrate oxidation rate was thought to be $\sim 1 \mathrm{~g} / \mathrm{min}$ (11). However, the co-ingestion of different carbohydrates may increase the oxidation rate further (for review see Jeukendrup (15)). When fructose is ingested together with glucose, exogenous oxidation rate can increase to $1.26 \mathrm{~g} / \mathrm{min}$ (12). With the co-ingestion of different carbohydrates, different intestinal transporters are used for absorption. Therefore, the capacity for total intestinal carbohydrates absorption increases during the co-ingestion of these multiple transportable carbohydrates. As a result, more carbohydrates can be absorbed by the intestinal transporters. The highest possible exogenous carbohydrate oxidation rate depends on the capacity of the intestinal transporters and on the number of carbohydrates that are available for absorption (9).

The ingestion of multiple transportable carbohydrates leads to a high exogenous carbohydrate oxidation and consequently to better performance compared to the ingestion of glucose $(16,17)$. Therefore, the experience of fatigue may be decreased following ingestion of multiple carbohydrates during a fixed workload. The hypothesis is that the ingestion of multiple transportable carbohydrates decreases the experience of fatigue further than a single transportable carbohydrate because the exogenous carbohydrate oxidation rate is higher.

In the present study, the hypothesis that the ingestion of multiple transportable carbohydrates as glucose with fructose or glucose with sucrose decreases the experience of fatigue compared to an isoenergetic amount of glucose was investigated. To investigate this hypothesis, 12 healthy, trained male cyclists participated to cycle 180 minutes at $50 \%$ of their Wmax while ingesting water, glucose $(1.8 \mathrm{~g} / \mathrm{min})$, glucose+fructose $(1.2+$ $0.6 \mathrm{~g} / \mathrm{min})$ or glucose+sucrose $(0.6+1.2 \mathrm{~g} / \mathrm{min})$. The subjects were asked to rate their perceived exertion every 30 minutes during exercise to assess their experience of fatigue. 
Furthermore, the subjects were asked to fill in a questionnaire every 30 minutes to assess gastrointestinal complaints. Breath-by-breath measurements were performed every 15 minutes to analyze substrate utilization.

\section{Material and methods}

\section{Subjects}

12 healthy, trained male cyclists participated in this study. Inclusion criteria were healthy, male, with an age between 18 and 40 years, endurance trained cyclist/triathlete, capable of producing Watt $/ \mathrm{kg}$ ffl 4.5 and a BMI lower than $25 \mathrm{~kg} / \mathrm{m}^{2}$. Subjects who use medication or tobacco were excluded from participation. The study was approved by the Medical Ethical Committee of the Maastricht University Medical Centre, Maastricht, The Netherlands.

\section{Preliminary testing}

The subjects were asked to fill in a medical questionnaire to determine their general health, use of medication, medical history and their sport activities for screening. One week before the start of the experimental trials, the subjects came to the lab for preliminary testing. During this meeting, the height and body weight of each subject was measured. In order to determine the individual maximum power output (Wmax) and peak oxygen uptake $\left(\mathrm{VO}_{\text {2peak }}\right)$, the subjects performed an incremental ergometer exercise test to volitional exhaustion. This test was performed on a cycle ergometer, modified to the configuration of a racing bicycle. The subjects first did a 5 minute warm-up at a workload of $100 \mathrm{~W}$, followed by incremental steps of $50 \mathrm{~W}$ every 2.5 minutes until exhaustion. The heart rate was recorded continuously by a heart rate monitor. The cadence was recorded at every interval. Breath-by-breath measurements were performed throughout exercise to assess the $\mathrm{O}_{2}$ uptake and the $\mathrm{CO}_{2}$ production using an online automated gas analysis system. The $\mathrm{VO}_{2 \max }$ was achieved when it met at least two of the three following criteria:1) a respiratory exchange ratio higher than 1.05, 2) a heart rate within 10 beats per minute of the maximal heart rate, and 3) a levelling off of $\mathrm{VO}_{2}$ with increasing workload. The maximum power output was calculated from the last completed work rate, plus the fraction of time spent in the final, non-completed work rate, multiplied by the work rate increment. The maximum power output was used to determine the work rate corresponding to $50 \% \mathrm{Wmax}$, which was later employed in the experimental trial.

\section{Experimental design}

During the experimental trial subjects were asked to cycle for 180 minutes at $50 \% \mathrm{Wmax}$, while receiving a glucose+fructose drink (GLU+FRUC), a glucose+sucrose drink (GLU+SUC), 
an isoenergetic glucose drink (GLU) or plain water (WAT). The carbohydrates used contained a high natural abundance of ${ }^{13} \mathrm{C}$ to quantify exogenous carbohydrate oxidation. The study was a double-blind, placebo-controlled study where the subjects were randomly assigned to a group.

\section{Diet and activity before testing}

48 hours prior to the experimental trial the subjects were asked to record their food intake and physical activity. The evening before the experimental trial, the subjects consumed a standardized meal as dinner. In addition, they were asked to perform an intense training session ('glycogen-depleting' exercise bout) 5 to 7 days before the experimental trial. The purpose of this was to empty any ${ }^{13} \mathrm{C}$-enriched glycogen stores. After this intense training session, the subjects were asked to refrain from any food products with a high natural abundance of ${ }^{13} \mathrm{C}$.

\section{Protocol}

The subjects arrived at the laboratory at 8:00 in the morning for the experimental trial after a 10 hour fast and having refrained from any strenuous activity or drinking any alcohol in the previous 24 hours. Prior to the commencement of the steady-state 180 minutes cycling exercise, a catheter was inserted in an antecubital vein of the subject's arm for repeated blood sampling, and the subjects were weighed and fitted with a heart rate monitor. In addition, they were familiarized with the Borg scale. The subjects were then seated on the cycle ergometer. A resting breath sample was collected in a $10-\mathrm{mL}$ Exetainer tube which were filled directly from a mixing chamber to determine the ${ }^{13} \mathrm{C} /{ }^{12} \mathrm{C}$ ratio in the expired air. A resting blood sample of $10 \mathrm{ml}$ was obtained, stored on ice and centrifuged. After this, the subjects exercised for 180 minutes at a work rate of $50 \%$ of their Wmax.

The ratings of perceived exertion of the subjects were determined during exercise using the Borg scale (18). Every 30 minutes the subjects were asked to rate their perceived exertion for the whole body on a scale from 6 to $20(6=$ no exertion at all, $7=$ extremely light, $9=$ very light, 11=light, 13=somewhat hard, 15= hard, 17=very hard, 19=extremely hard and $20=$ maximal exertion) (figure 1 ). The ratings of perceived exertion indicate how heavy and strenuous the exercise feels, combining all sensations and feelings of fatigue, effort and physical stress. 
Gastrointestinal complaints were also monitored by having the subjects fill in a questionnaire during exercise (figure 1). The questionnaire addressed the presence of complaints of gastrointestinal problems at that moment. These complaints were nausea, full feeling, urge to vomit, headache, dizziness, bloated feeling, flatulence, diarrhea, obstipation, urge to urinate, urge to defecate, belching, discomfort in gastric region, stomach burn, stomach cramps and intestinal cramps. Every 30 minutes the subjects were asked to give a number on a 10-point scale that corresponded to the severity of the complaint, with 1 corresponding to not at all and 10 to very, very much.

The RER, $\mathrm{VO}_{2}$ and $\mathrm{VCO}_{2}$ were assessed every 15 minutes for periods of 4 minutes using an online automated gas analysis system. Expiratory breath samples were collected every 15 minutes during exercise. Blood samples were drawn every 30 minutes during exercise (figure 1).

During the first 3 minutes of exercise, the subjects drank $600 \mathrm{~mL}$ of one of the four experimental drinks (WAT, GLU, GLU+FRUC or GLU+SUC). Thereafter, $150 \mathrm{~mL}$ of the experimental drink was ingested every 15 minutes until the end of the exercise $(T=180)$ (figure 1). The total fluid that was ingested during the 180 minutes of exercise was 2.25 liter. The total carbohydrate ingestion of each drink was 1.8 gram per minute. The GLU consisted of 1.8 gram glucose per minute, the GLU+FRUC consisted of 1.2 gram glucose + 0.6 gram fructose per minute and the GLU+SUC consisted of 0.6 gram glucose +1.2 gram sucrose per minute. $20 \mathrm{mmol} \cdot \mathrm{L}^{-1}$ of sodium chloride was added to all of the drinks.

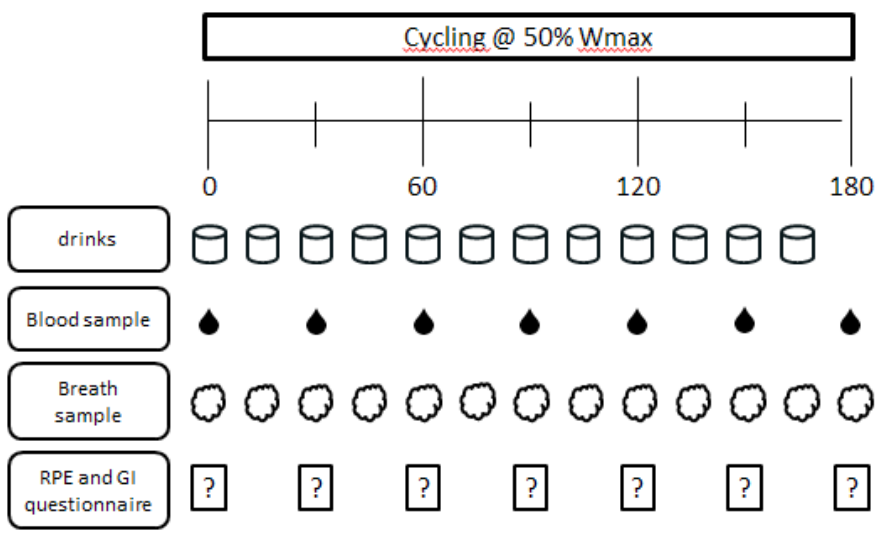

Figure 1. Experimental design 


\section{Analyses}

Breath samples were analyzed for ${ }^{13} \mathrm{C} /{ }^{12} \mathrm{C}$ ratio by gas chromatography continuous flow isotope ratio mass spectrometry (GC-IRMS). Blood samples were collected in EDTA containing tubes and centrifuged at $3000 \mathrm{~g}$ and $4^{\circ} \mathrm{C}$ for 10 minutes. $2 \mathrm{ml}$ of plasma was added in 5 Eppendorf tubes, frozen in liquid nitrogen and stored at $-80 \mathrm{C}$ until analysis of glucose, lactate and insulin by radio immunoassay.

The formulas which follow were used to calculate total carbohydrate oxidation, fat oxidation and exogenous carbohydrate rates. The $\mathrm{VCO}_{2}$ and $\mathrm{VO}_{2}$ from the indirect calorimetry were used to calculate total fat and total carbohydrate oxidation following the stoichiometri equations of Frayn (19).

\section{Formulas:}

- Total $\mathrm{CHO}$ oxidation $=4.55 \mathrm{VCO} 2-3.21 \mathrm{VO} 2$

- Fat oxidation $=1.67 \mathrm{VO} 2-1.67 \mathrm{VCO} 2$

- Exogenous carbohydrate oxidation $=\mathrm{VCO} 2 \cdot(\delta$ Exp $-\delta$ Expbkg $) /(\delta \mathrm{Ing}-\delta$ Expbkg $)-1 / \mathrm{k}$

\section{Statistical analyses.}

Data for subject characteristics such as age, weight, height, BMI, Wmax, Wmax/kg and $\mathrm{VO}_{\text {2peak }}$ were presented as means \pm standard error. A one-way analysis of variance (ANOVA) was used to compare the RPE between the four drinks at one time point. A mixed ANOVA was used to determine the effect of the different drinks on the RPE, indirect calorimetry and exogenous carbohydrate oxidation over time. A Tukey post hoc test was applied to locate differences when ANOVA revealed a significant interaction. The statistical significance was set at $\mathrm{P}<0.05$ for all tests. Data evaluation was performed using SPSS.

\section{Results}

The results of this study cannot be published.

\section{Discussion/Conclusion}

The results of the study cannot be published but speculations can be made. Other studies have shown no significant difference in the mean RPE when a glucose drink was compared with a glucose+sucrose+fructose drink, a glucose+fructose drink or a glucose+fructose drink $(9,13)$. While studies showed no difference in mean RPE between carbohydrate drinks and water, several studies did show a difference during the latter stages of exercise $(3,6,7$, 20). The same conclusions can be expected in this study: there will probably be significant 
differences in RPE between the four drinks during later stages of exercise. Endogenous carbohydrate substrates provide energy at the start of exercise, thus RPE is expected to be the same for all groups when exercise starts. As exercise continues, endogenous carbohydrate energy substrates such as glycogen decrease significantly (20). As a result, a localized muscular fatigue is induced as the energy supply to the myofibril contraction is interrupted. Carbohydrates will most likely minimize this localized muscular fatigue, as they provide exogenous carbohydrate energy substrates to glycogen-depleted muscle fibers (2). Carbohydrate ingestion may therefore lower RPE during the latter stages of exercise compared to the water trial.

RPE is expected to be lower when multiple transportable carbohydrates are ingested compared to the glucose trial. With the ingestion of multiple transportable carbohydrates, different intestinal transporters are used for absorption and therefore more carbohydrates can be absorbed. As a result, the exogenous carbohydrate oxidation increases, which will increase performance. When exercising at a fixed workload, ingesting multiple transportable carbohydrates are expected to achieve an RPE lower than those achieved by the ingestion of glucose.

The ingestion of carbohydrates is likely to reduce the experience of fatigue compared to the ingestion of water only. This speculation is based on the fact that the rate of total carbohydrate oxidation is probably higher and maintained with the ingestion of carbohydrates and especially with the ingestion of multiple transportable carbohydrates (3). With the large supply of carbohydrates, the blood glucose concentration is maintained. Blood glucose is an important source of energy for working muscles and can still supply energy when carbohydrates are ingested. When only water is ingested, the glycogen levels are low late in exercise and there is no supply of energy to the working muscles, therefore the experience of fatigue is expected to be higher.

More speculations can be made about the gastrointestinal complaints. One such speculation is that there will be high gastrointestinal complaints when glucose is ingested, as glucose is a single transportable carbohydrate. Gastrointestinal complaints can be experienced when a large amount of single transportable carbohydrates is ingested $(12,13)$, because there will be an incomplete absorption as a result of lower intestinal carbohydrate transport capacity (13). The carbohydrates accumulate in the gastrointestinal tract as fewer carbohydrates are absorbed by the intestinal transporters. The resulted complaints may include nausea, bloated feeling, full feeling, belching and discomfort in the gastric 
region. The prevalence of these complaints can be lower when multiple transportable carbohydrates are ingested because of a faster carbohydrate absorption rate. Less carbohydrate accumulates in the Gl tract with the ingestion of multiple transportable carbohydrates compared to glucose only. As gastrointestinal complaints increase, it is expected that performance will be adversely affected (5). Therefore, the RPE is likely to be higher in the glucose trial compared to the trials with ingestion of multiple transportable carbohydrates.

In summary, when glucose and fructose or glucose and sucrose are ingested simultaneously at high rates during endurance exercise, the experience of fatigue is likely to be reduced compared with the ingestion of an isoenergetic amount of glucose.

\section{Role of the student}

Lonneke Albert was an internship student working under the supervision of PhD student J. Trommelen when the research in this report was performed. The topic was proposed by the student. The student was responsible for the processing of the results, formulation of the conclusion and the writing. The study design was invented by the supervisor and both the student and supervisor participated in data collection.

\section{Acknowledgments}

I would like to thank my supervisor J. Trommelen for his helpful guidance during my internship. He taught me about performing research and provided many helpful comments during the writing of my report. I would also like to thank all of the other colleagues that helped with the collection of data.

\section{References}

1. Edwards RH. Human muscle function and fatigue. Human muscle fatigue: physiological mechanisms. 82: Pitman Medical London; 1981. p. 1-18.

2. Ament W, Verkerke GJ. Exercise and fatigue. Sports Medicine. 2009;39(5):389-422.

3. Burgess ML, Robertson RJ, Davis JM, Norris JM. RPE, blood glucose, and carbohydrate oxidation during exercise: effects of glucose feedings. Medicine and science in sports and exercise. 1991;23(3):353-9.

4. Coyle EF, Coggan AR, Hemmert MK, Ivy JL. Muscle glycogen utilization during prolonged strenuous exercise when fed carbohydrate. Journal of applied physiology (Bethesda, Md : 1985). 1986;61(1):165-72. 
5. Rowlands DS, Thorburn MS, Thorp RM, Broadbent S, Shi X. Effect of graded fructose coingestion with maltodextrin on exogenous $14 \mathrm{C}$-fructose and $13 \mathrm{C}$-glucose oxidation efficiency and high-intensity cycling performance. Journal of applied physiology (Bethesda, Md : 1985). 2008;104(6):1709-19.

6. Utter AC, Kang J, Nieman DC, Williams F, Robertson RJ, Henson DA, et al. Effect of carbohydrate ingestion and hormonal responses on ratings of perceived exertion during prolonged cycling and running. Eur J Appl Physiol Occup Physiol. 1999;80(2):92-9.

7. Backhouse SH, Bishop NC, Biddle SJ, Williams C. Effect of carbohydrate and prolonged exercise on affect and perceived exertion. Medicine and science in sports and exercise. 2005;37(10):1768-73.

8. Jeukendrup AE. Carbohydrate intake during exercise and performance. Nutrition. 2004;20(7-8):669-77.

9. Jentjens RL, Jeukendrup AE. High rates of exogenous carbohydrate oxidation from a mixture of glucose and fructose ingested during prolonged cycling exercise. The British journal of nutrition. 2005;93(4):485-92.

10. Jeukendrup A. A step towards personalized sports nutrition: carbohydrate intake during exercise. Sports medicine (Auckland, NZ). 2014;44 Suppl 1:S25-33.

11. Jeukendrup AE, Jentjens R. Oxidation of carbohydrate feedings during prolonged exercise: current thoughts, guidelines and directions for future research. Sports medicine (Auckland, NZ). 2000;29(6):407-24.

12. Jentjens RL, Moseley L, Waring RH, Harding LK, Jeukendrup AE. Oxidation of combined ingestion of glucose and fructose during exercise. Journal of applied physiology (Bethesda, Md : 1985). 2004;96(4):1277-84.

13. Jentjens RL, Achten J, Jeukendrup AE. High oxidation rates from combined carbohydrates ingested during exercise. Medicine and science in sports and exercise. 2004;36(9):1551-8.

14. Davidson RE, Leese HJ. Sucrose absorption by the rat small intestine in vivo and in vitro. J Physiol. 1977;267(1):237-48.

15. Jeukendrup AE. Carbohydrate feeding during exercise. European Journal of Sport Science. 2008;8(2):77-86.

16. Currell K, Jeukendrup AE. Superior endurance performance with ingestion of multiple transportable carbohydrates. Medicine and science in sports and exercise. 2008;40(2):275-81.

17. Triplett D, Doyle JA, Rupp JC, Benardot D. An isocaloric glucose-fructose beverage's effect on simulated $100-\mathrm{km}$ cycling performance compared with a glucose-only beverage. Int J Sport Nutr Exerc Metab. 2010;20(2):122-31.

18. Borg GA. Psychophysical bases of perceived exertion. Medicine and science in sports and exercise. 1982;14(5):377-81.

19. Frayn KN. Calculation of substrate oxidation rates in vivo from gaseous exchange. J Appl Physiol Respir Environ Exerc Physiol. 1983;55(2):628-34.

20. Kang J, Robertson RJ, Goss FL, DaSilva SG, Visich P, Suminski RR, et al. Effect of carbohydrate substrate availability on ratings of perceived exertion during prolonged exercise of moderate intensity. Percept Mot Skills. 1996;82(2):495-506. 